\title{
Atuação da enfermagem na Saúde do Idoso: perspectivas de ações intersetoriais e multiprofissionais para a melhoria do estilo de vida
}

\author{
Nursing performance in Elderly Health: perspectives of intersectoral and multiprofessional actions \\ for improving lifestyle
}

Desempeño de enfermería en salud del anciano: perspectivas de acciones intersectoriales y multiprofesionales para mejorar el estilo de vida

Recebido: 27/12/2020 | Revisado: 28/12/2020 | Aceito: 01/01/2021 | Publicado: 02/01/2021

\author{
Maria de Fátima Santana de Souza Guerra \\ ORCID: https://orcid.org/0000-0002-2760-8230 \\ Centro Universitário UniAges, Brasil \\ E-mail: marinaide10@gmail.com \\ Ana Mara Borges Araujo \\ ORCID: https://orcid.org/0000-0003-4065-241X \\ Centro Universitário UniAges, Brasil \\ E-mail: anamaraborges_@hotmail.com \\ Jaciara Pinheiro de Souza \\ ORCID: https://orcid.org/0000-0002-4056-974X \\ Centro Universitário UniAges, Brasil \\ E-mail: jacipinheirosouza@hotmail.com \\ Murilo de Jesus Porto \\ ORCID: https://orcid.org/0000-0003-2339-8173 \\ Universidade do Estado da Bahia, Brasil \\ E-mail: murilo.porto@ hotmail.com \\ Maciel Borges Nascimento \\ ORCID: https://orcid.org/0000-0002-9190-6072 \\ Centro Universitário UniAges, Brasil \\ E-mail: macielborges17@gmail.com \\ Walber Barbosa de Andrade \\ ORCID: https://orcid.org/0000-0003-0290-5009 \\ Centro Universitário UniAges, Brasil \\ E-mail: blekwalber@hotmail.com \\ Welde Natan Borges de Santana \\ ORCID: https://orcid.org/0000-0001-9781-2934 \\ Centro Universitário UniAges, Brasil \\ E-mail:welder_natan@hotmail.com \\ Gilvania Piedade Santos \\ ORCID: https://orcid.org/0000-0003-2435-0198 \\ Centro Universitário UniAges, Brasil \\ E-mail: gilvania.piedade@gmail.com \\ Antero Fontes de Santana \\ ORCID: https://orcid.org/0000-0002-8944-3216 \\ Centro Universitário UniAges, Brasil \\ E-mail: anterosantana@ @otmail.com \\ Sandra Regina Santana Silva \\ ORCID: https://orcid.org/0000-0002-8218-3407 \\ Centro Universitário UniAges, Brasil \\ E-mail: sandrareginaenfa@hotmail.com
}

\begin{abstract}
Resumo
A Política Nacional de Promoção da Saúde apontava para a explicitação de mecanismos que reduziriam as situações de vulnerabilidade, defendiam radicalmente a equidade e incorporavam a participação e o controle social na gestão das políticas públicas, assim houve o aumento no número de idosos, dentre outros fatores, necessitando a retomar as discussões que permeiam a crise no setor da saúde. Este estudo tem como objetivo discutir a atuação do profissional de enfermagem na prestação de assistência à saúde do idoso em uma perspectiva de ações intersetoriais e multiprofissionais para a melhoria do estilo de vida desse público. A metodologia é de revisão narrativa, através de levantamento bibliográfico em fontes seguras de dados, sem restrição de ano de publicação dos trabalhos. Como resultados apresentados, é mostrado que o enfermeiro é extremamente importante na equipe multiprofissional nas unidades de saúde no desenvolvimento de ações de prevenção, promoção e recuperação da saúde do idoso, além da
\end{abstract}


prevenção de doenças. Mediante levantamento bibliográfico, conclui-se para que se tenha uma atenção qualificada, resolutiva e eficaz aos idosos, é necessária a formação de profissionais, entre estes o enfermeiro, devidamente qualificados para vislumbrar a tenacidade da instalação de processos patológicos nos idosos, os quais por muitas vezes pode mudá-los de independente para dependente.

Palavras-chave: Envelhecimento; Saúde do idoso; Sociedade.

\begin{abstract}
The National Health Promotion Policy pointed to the explanation of mechanisms that would reduce situations of vulnerability, radically defend equity and incorporate participation and social control in the management of public policies, thus there was an increase in the number of elderly people, among other factors, needing to resume the discussions that permeate the crisis in the health sector. This study aims to discuss the role of the nursing professional in providing health care to the elderly in a perspective of intersectoral and multiprofessional actions to improve the lifestyle of this public. The methodology is of narrative review, through bibliographic survey in secure data sources, without restriction of year of publication of the works. As presented results, it is shown that nurses are extremely important in the multiprofessional team in health units in the development of prevention, promotion and recovery actions for the health of the elderly, in addition to disease prevention. Through a bibliographic survey, it is concluded that in order to have a qualified, resolutive and effective attention to the elderly, it is necessary to train professionals, including nurses, duly qualified to glimpse the tenacity of the installation of pathological processes in the elderly, you can often change them from independent to dependent.
\end{abstract}

Keywords: Aging; Elderly health; Society.

\title{
Resumen
}

La Política Nacional de Promoción de la Salud apuntó a la explicación de mecanismos que reduzcan situaciones de vulnerabilidad, defiendan radicalmente la equidad e incorporen la participación y el control social en la gestión de las políticas públicas, por lo que hubo un aumento en el número de personas mayores, entre otros factores, necesitando retomar las discusiones que permean la crisis en el sector salud. Este estudio tiene como objetivo discutir el papel del profesional de enfermería en la prestación de cuidados de salud a las personas mayores en una perspectiva de acciones intersectoriales y multiprofesionales para mejorar el estilo de vida de este público. La metodología es de revisión narrativa, mediante levantamiento bibliográfico en fuentes de datos seguras, sin restricción de año de publicación de los trabajos. Como se presentan los resultados, se demuestra que el enfermero es de suma importancia en el equipo multiprofesional de las unidades de salud en el desarrollo de acciones de prevención, promoción y recuperación de la salud de las personas mayores, además de la prevención de enfermedades. A través de un relevamiento bibliográfico, se concluye que para tener una atención calificada, resolutiva y eficaz al anciano, es necesario formar profesionales, incluidos enfermeros, debidamente capacitados para vislumbrar la tenacidad de la instalación de procesos patológicos en el anciano, a menudo puede cambiarlos de independientes a dependientes.

Palabras clave: Envejecimiento; Salud de los ancianos; Sociedad.

\section{Introdução}

Pode-se notar o declínio da fecundidade nos países em desenvolvimento, reduzindo assim o número de jovens e contribuindo para a caracterização de um perfil populacional envelhecido (Brasil, 2010). Dessa forma, pode-se perceber o impacto social, de modo que aumenta os custos do Instituto Nacional Seguro Social - INSS, atendimento de saúde e construção de asilos. E estes idosos, na maioria das vezes, não possuem cuidadores ou parentes que prestem assistência adequada, ideia que contradiz a beleza da longevidade, ou seja, a esperança de vida ao nascer, a expectativa de crescer, reproduzir e acompanhar suas gerações futuras (Siqueira et al., 2008).

Assim sendo, os dados epidemiológicos, enfatizam o envelhecimento global e demonstram a expectativa do aumento de vida de dois bilhões de pessoas com sessenta anos ou mais para 2050. Desse modo, pode-se perceber o resultado da maior atenção da Organização Mundial de Saúde - OMS e do Ministério da Saúde - MS às políticas de saúde a pessoa idosa tendo como resposta as mudanças nos indicadores de saúde, bem como o aumento progressivo desse grupo (Brasil, 2010 \& Matsudo, 2001).

Sugere-se que o melhor investimento na atenção primária, tanto em programas geriátricos quanto em políticas sociais gerais, promoverá o bem-estar dos que estão no estado de senescência, de modo que atividades e exercícios físicos regulares, cotidianamente colaboram na capacidade funcional, instrumental e autonomia das atividades de vida diárias. Sendo estas influenciadas por meio dos profissionais de saúde qualificados, bem como incentivados por seus gestores os quais devem 
oferecer recursos humanos para equipe, de modo que tais atitudes venham representar impacto social positivo na melhoria dos índices de saúde populacional tanto por ser um custo financeiro menor, quanto por promover estilos de vida mais saudáveis e qualidade na saúde do idoso (Joia et al., 2013 \& Silva et al., 2012 \& Siqueira et al., 2008 \& Pereira, 2006).

Nesse sentido, essa pesquisa voltou-se para um levantamento de dados bibliográficos, com o objetivo de discutir a atuação do profissional de enfermagem na prestação de assistência à saúde do idoso em uma perspectiva de ações intersetoriais e multiprofissionais para a melhoria do estilo de vida desse público.

\section{Metodologia}

Trata-se de uma revisão narrativa, realizada por meio de levantamento bibliográfico, sendo caracterizada como:

A revisão bibliográfica é indispensável para a delimitação do problema em um projeto de pesquisa e para obter uma ideia precisa sobre o estado atual dos conhecimentos sobre um tema, sobre suas lacunas e sobre a contribuição da investigação para o para o desenvolvimento do conhecimento para (Lakatos \&Marconi, 2010, p.25).

Assim, a presente pesquisa possui característica descritiva, possuindo uma descrição das propriedades de uma determinada população ativa no momento da pesquisa, conforme Gil (2012), tendo por desígnio mostrar as opiniões e créditos. Admite, em geral, a configuração de classificação dos devidos dados.

A pesquisa exploratória analisa, através de uma problemática, uma visão ampla e mostra quesitos explícitos visando promover a edificação de hipóteses. Esse tipo de pesquisa tem como fundamental desígnio o descobrimento de novas ideias (Gil, 2011).

Uma pesquisa de caráter bibliográfico desenvolve suas análises através de objetivos, justificativa e problemática que envolve vários níveis de informações para englobar aspectos essenciais do conhecimento de determinado tema e discutir com distintos autores a devida pesquisa, mostrando as considerações finais (Gil, 2011).

Desta forma, a devida pesquisa envolve o estudo sobre a atuação da enfermagem na assistência ao idoso, sendo analisado, na primeira etapa, a temática e os assuntos de interesse; na segunda etapa ocorreu a consignação dos discernimentos de inclusão e exclusão da pesquisa; já na terceira etapa foi englobada a acepção dos subsídios a serem adquiridas e classificação dos estudos; a quarta etapa, foi desenvolvida a análise dos resultados e na quinta etapa: a avaliação de todos os resultados, almejando atingir os objetivos da pesquisa e interpretação dos achados.

Os critérios de inclusão utilizados nesse trabalho foram artigos completos e publicados no idioma português; obras publicadas na íntegra e que fossem do tema estudado; sites confiáveis; cadernos da atenção básica dirigidos aos idosos; não houve restrição de ano de publicação. Os critérios de exclusão foram artigos ou obras afins repetidos e incompletos; resenhas; relatos de casos; artigos publicados em anais de eventos; obras que cobrassem pelo acesso.

\section{Resultados e Discussão}

\section{O Papel do Enfermeiro na Saúde do Idoso}

Historicamente, a atenção à saúde no Brasil tem investido na formulação, implementação e concretização de políticas de promoção, proteção e recuperação da saúde. Há, pois, um grande esforço na construção de um modelo de atenção à saúde que priorize ações de melhoria da qualidade de vida dos sujeitos e do coletivo. Nessa direção, o desafio colocado para o gestor federal do SUS consistiu em propor uma política transversal, integrada e intersetorial, que proporcionasse o diálogo entre as diversas áreas do setor sanitário, os outros setores do Governo, os setores privado e não governamental e a sociedade, compondo redes de compromisso e corresponsabilidade quanto à qualidade de vida da população em que todos sejam partícipes no cuidado com a saúde (Brasil, 2010). 
O ano de 2006 foi particularmente marcante em relação às práticas que o Brasil desenvolve para integralidade do cuidado e para a melhoria da qualidade de vida da população. Nesse ano foi aprovada a Política Nacional de Promoção da Saúde - PNPS, pela portaria ${ }^{\circ}$ 687, a qual mostra a Promoção da Saúde entendida como uma estratégia de articulação transversal que busca promover a qualidade de vida e reduzir a vulnerabilidade e os riscos à saúde relacionados aos seus determinantes e condicionantes - modos de viver, condições de trabalho, habitação, ambiente, educação, lazer, cultura, acesso a bens e serviços essenciais (Brasil, 2010).

A Política Nacional de Promoção da Saúde apontava para a explicitação de mecanismos que reduzam as situações de vulnerabilidade, defendiam radicalmente a equidade e incorporavam a participação e o controle social na gestão das políticas públicas. Definiam ainda, a importante interface com a Atenção Básica e a Estratégia Saúde da Família, lócus de execução dessas diretrizes. Assim, a nova Política Nacional de Promoção da Saúde priorizava ações voltadas para a divulgação e implementação dessa Política. Neste contexto estão: alimentação saudável; prática corporal/ atividade física; prevenção e controle do tabagismo; redução da morbimortalidade em decorrência do uso abusivo de álcool e outras drogas; redução da morbimortalidade por acidente de trânsito; prevenção da violência e estímulo à cultura de paz; promoção do desenvolvimento sustentável (Brasil, 2010 \& Silva, 2012).

Sabe-se que o envelhecimento é um processo multifatorial, desse modo se faz necessário enfatizar os fatores de prevenção primária os quais se referem à atenção aos riscos ambientais ou pessoais que possam ser desencadeadores de doenças e não podem ser alterados, como idade, etnia e fatores genéticos. Assim, é possível que o envelhecimento saudável seja conquistado por indivíduos que desde a juventude, ou antes, tenham se preocupado em fazer uso de uma dieta adequada e de práticas esportivas, não se tornar dependente químico ou de fatores debilitantes, como alto nível de estresse físico e mental. Em outras palavras, a qualidade de vida é imperativa para um bom envelhecimento (Brasil, 2007).

Neste aspecto é fundamental que o profissional, assim como os próprios idosos, seus familiares e cuidadores tenham uma visão integrada destes fenômenos. O enfermeiro é ator principal na atenção primária educando a população jovem desde a infância à fase adulta a adotarem estilos de vida saudáveis, além de orientar os seus idosos a melhorarem os hábitos de vida na perspectiva de uma melhor qualidade de vida e longevidade saudável (Achutti, 2004).

O enfermeiro é extremamente importante na equipe multiprofissional da Estratégia Saúde da Família- ESF no desenvolvimento de ações de prevenção, promoção e recuperação da saúde do idoso, além da prevenção de doenças. Além disso, este profissional facilita o atendimento de identificação de pontos de intervenção para com o idoso exemplificado a partir dos instrumentos de Sistematização da Assistência de Enfermagem - SAE (Tanure, 2010).

A equipe de enfermagem precisa reconhecer sua importância como educadora e agente de transformação social que necessita ser constante em sua atuação profissional, já que parte integrante de sua designação profissional deve ser a educação permanente em saúde, isso porque é através da enfermagem que a interação entre profissionais e usuários do sistema de saúde é feita. Esse profissional deve refletir de forma consciente sobre suas ações e atitudes no cuidado com o idoso, ele precisa possibilitar o desenvolvimento de práticas holísticas, que tragam alguma reflexão e sejam capazes de auxiliar a assistência do cuidador (Brasil, 2007).

Mediante as necessidades do idoso as principais funções do enfermeiro em uma rotina de ações sistematizada ao idoso realizado na ESF são: avaliação geronto - geriátrica, com base nos passos da SAE, avaliando perfil nutricional, psicológico, sócio - econômico, sócio - cultural, ambiente, moradia; avaliação de Atividade de Vida Diária AVD; exames laboratoriais; imunizações; tratamento medicamentoso e não medicamentoso; saúde bucal; trabalho em grupo; visitas domiciliares (Brasil, 2010). 


\section{Assistência do idoso na Estratégia de Saúde da Família}

A Estratégia de Saúde da Família (ESF) nada mais é que uma unidade de serviços públicos de saúde voltada a realizar uma atenção sucessiva nas especialidades de análises básicas com uma equipe multidisciplinar habilitada aumentando as atividades de níveis primários incluindo a rede de promoção, proteção e recuperação (Figueiredo, 2015).

Segundo a visão de alguns educadores a EPS constitui uma abertura do movimento institucionalista em educação, ligando-se como o elemento humano nas organizações. Sob o ponto de vista é sugerida a gestão dos coletivos e a criação de amplificadores para que cada grupo humano se reúna e dialoga-se seus problemas do cotidiano, facilitando a ampliação significativa da aprendizagem em cada tempo e lugar (Ceccim, 2005).

Com isto essa ESF concebe o primeiro acionador da população com o serviço de saúde do município, certificando a referência os âmbitos de contra referência para os diferentes níveis do sistema, desde que esteja coligada na análise do objetivo a necessidade de maior complexidade tecnológica para a licença dos problemas identificados da saúde (Figueiredo, 2015).

A educação em saúde em geral é a concretização do encontro entre o mundo de desenvolvimento de formação da população e o mundo das atividades trabalhistas, onde o aprender e o ensinar se interligam ao cotidiano das disposições de maneira geral (Brasil, 2009).

As ESF caracterizam-se como uma porta de entrada da referência local de saúde, onde não significa somente a criação de novos alicerces de caráter assistenciais, exceto em áreas desprovidas, mas supre as práticas essenciais pela oferta de um desempenho centrado nos regimentos da vigilância à saúde (Figueiredo, 2015).

$\mathrm{O}$ aumento no número de idosos, dentre outros fatores, suscita a necessidade de retomar as discussões que permeiam a crise no setor da saúde. Nesta perspectiva, visando programar os princípios e diretrizes do Sistema Único de Saúde - SUS, o Ministério da Saúde - MS institui a ESF, em 1994. Tal estratégia enfoca a família como unidade de ação programática de saúde e não mais, tão somente o indivíduo, logo que a Atenção Básica se caracteriza por um conjunto de ações de saúde, no âmbito individual e coletivo, que abrange a promoção, a proteção, a prevenção, o diagnóstico, o tratamento, a reabilitação e a manutenção da saúde. Ela acontece por meio das práticas gerenciais e sanitárias - democráticas e participativas -, sob a forma de trabalho em equipe, dirigida a populações de territórios bem delimitados, pelas quais assume a responsabilidade sanitária, considerando a dinamicidade existente no espaço em que vivem (Carvalho, 2006).

A ESF está pautada na visão ativa da intervenção em saúde, ou seja, não somente esperar a população chegar aos serviços de saúde para intervir. Deve-se interagir com ela preventivamente, constituindo-se em instrumento real de reorganização de demanda. Além disso, reforçam-se as concepções de integração com a comunidade e o enfoque na atenção integral, evitando ações reducionistas em saúde, centradas, somente, na intervenção biológica e médica. Neste contexto, há uma indução do Governo brasileiro para que o setor saúde reconstrua a produção em saúde. Visa-se, então, qualificar os profissionais, corresponsabilizá-los pelo cuidado prestado e estimular a atenção integral à saúde de todos os membros familiares, nas diversas fases do ciclo vital (Brasil, 2012).

$\mathrm{O}$ aumento da população idosa, o que vem ocorrendo de forma rápida e progressiva, exige que os profissionais da saúde, em especial os enfermeiros, estejam capacitados para atender as especificidades desta etapa da vida, melhorando a assistência prestada (Carvalho, 2006).

A Política Nacional de Saúde do Idoso, instituída em 1999, tem como propósito basilar a promoção do envelhecimento saudável; a manutenção e a melhoria, ao máximo, da capacidade funcional dos idosos; a prevenção de doenças, a recuperação da saúde e a reabilitação. Busca-se garantir a permanência do idoso no meio em que vive, exercendo de forma independente suas funções na sociedade (Brasil, 2003).

Espera-se, portanto, que a formação acadêmica de profissionais enfermeiros seja baseada na perspectiva de desenvolver atividades, que não apenas informem sobre o processo de envelhecimento, mas que formem profissionais 
sensíveis aos limites e peculiaridades presentes nos idosos, a fim de compreender as modificações físicas, emocionais e sociais desta faixa etária (Carvalho, 2006). Além disso, as consultas de enfermagem necessitam ser implementadas nos serviços de saúde, uma vez que favorece o trabalho multiprofissional, o desenvolvimento de práticas intersetoriais, o relacionamento interpessoal com cliente e familiares e o cuidado baseado na cientificidade (Ms, 2012).

A ESF constitui-se em espaço privilegiado para atenção integral à saúde do idoso, pois sua proximidade com a comunidade e a atenção domiciliária possibilita atuar de forma contextualizada na realidade vivenciada pelo idoso no seio familiar. A efetiva inserção do idoso em Unidades de Saúde, sobretudo aquelas sob a ESF, pode representar para ele o vínculo com o sistema de saúde (OLIVEIRA, 2010).

A educação em saúde em âmbito de contexto é um assunto importante e categórico da qualidade de assistência em saúde, sendo abordada como aspectos de atualização técnico-científica de caráter contínuo onde ofertar aos profissionais um determinado pensamento de atuação prática, promovendo o alargamento de atuação na determinada instituição, mostrando, a experiência abordada e aprendida naquela capacitação para assim, poder atuar no desempenho profissional (Sardinha-Peixoto et al., 2013).

Considerando as especificidades do processo de envelhecimento e a necessária adequação e qualificação profissional, assim como, as possíveis lacunas na formação do profissional de saúde, o presente estudo visa contribuir na discussão, reflexão e reorganização das ações dos enfermeiros na atenção à saúde da população idosa, no âmbito da ESF (Leopardi, 2000).

\section{Considerações Finais}

Mediante levantamento bibliográfico, conclui-se para que se tenha uma atenção qualificada, resolutiva e eficaz aos idosos, é necessária a formação de profissionais, entre estes o enfermeiro, devidamente qualificados para vislumbrar a tenacidade da instalação de processos patológicos nos idosos, os quais por muitas vezes pode mudá-los de independente para dependente.

Vale ressaltar que a estratégia saúde da família tem importante atribuição na manutenção da qualidade de vida do idoso por meio de sua equipe sendo o enfermeiro principal responsável pelas práticas de cuidado devendo obter conhecimento para uma atenção que abranja os aspectos biopsicossociais. O alcance desse profissional é amplificado em virtude do vínculo de confiança com a comunidade e este deve agir como agente educacional, social na identificação e busca ativa dos idosos para prevenção de doenças e agravos comuns nessa faixa etária e promoção de um envelhecimento saudável no território assistido.

No cenário futuro, essa revisão bibliográfica contribuirá na ênfase da equipe do programa saúde da família, em especial o enfermeiro, como o alicerce na identificação, busca ativa, proteção e promoção da qualidade de vida dos idosos com foco no cuidado disponibilizado no seu local de vivência. Além disso, esse trabalho se configura como uma fonte de pesquisa na implementação de práticas de saúde voltadas ao idoso e promoção de embasamento científico em discussão pertinentes ao tema.

\section{Referências}

Achutti, A., \& Azambuja, M. I. R. (2004) Doenças crônicas não transmissíveis no Brasil: repercussões do modelo de atendimento à saúde sobre a seguridade social. http://www.scielo.br/pdf/csc/v9n4/a02v9n4.pdf.

Brasil. (2010). Envelhecimento e saúde da pessoa idosa. Série A. Normas e Manuais Técnicos Cadernos de atenção básica, 19. Brasília - DF.

Lei $\mathrm{N}^{\circ}$ 5095/73, de 12 de julho de 1973. (1973) Dispõe a criação do Conselho Federal e Conselho Regional de enfermagem e dá outras providencias. <http://presrepublica.jusbrasil.com.br/legislacao/128479/lei-5905-73>.

Ministério da Saúde (2003). Estatuto do Idoso. Brasília: Ministério da Saúde.

Ministério da Saúde (2013). Revista Brasileira Saúde da Família No33 /23 de abril. Ministério da Saúde - Ano 13, n. 33(2012). Brasília: Ministério da Saúde, 2012 . 
Research, Society and Development, v. 10, n. 1, e11210111536, 2021

(CC BY 4.0) | ISSN 2525-3409 | DOI: http://dx.doi.org/10.33448/rsd-v10i1.11536

Ministério da Saúde (2006). Revista Brasileira Saúde da Família Ministério da Saúde Secretaria de Atenção à Saúde Departamento de Atenção Básica. Brasília.

Ministério da Saúde. Política Nacional de Educação Permanente em $\quad$ Saúde. 9 , Brasília, http://portal.anvisa.gov.br/documents/33856/396770/Pol\%C3\%ADtica+Nacional+de+Educa\%C3\%A7\%C3\%A3o+Permanente+em+Sa\%C3\%BAde/c92db117 -e170-45e7-9984-8a7cdb111faa.

Ceccim, R. B. (2005). Educação Permanente em Saúde: desafio ambicioso e necessário. Revista Interface (Botucatu), Botucatu, 9(16).

Carvalho, Y. M. (2006). Promoção da Saúde, Práticas Corporais e Atenção Básica. USP São Paulo.

Figueiredo, E. N. Estratégia Saúde da Família e Núcleo de Apoio à Saúde da Família: diretrizes e fundamentos. Módulo Político Gestor, UNASUS, unifesp, 2015. http://www.unasus.unifesp.br/biblioteca_virtual/esf/1/modulo_politico_gestor/Unidade_5.pdf.

Gil, A. C. (2011). Método e Técnicas de Pesquisa Social. (6a ed.), São Paulo: Atlas, 2011.

Joia, L. C., et al. (2013). Condições associadas ao grau de satisfação com a vida entre a população de idosos. Cad. Saúde Pública. 29(1).

Leopardi, M. T., Santos, I., \& Sena, R. R. (2000). Tendências de enfermagem no Brasil: tecnologias do cuidado e valor da vida. Anais 51 CBEn, $10^{\circ}$ Congresso Panamericano de Enfermería. ABEn. 147-73.

Lakatos, E. M., \& Marconi, M. A. (2010). Fundamentos da metodologia científica. (7a ed.), São Paulo: Atlas.

Matsudo, S. M. (2001). Atividade física e envelhecimento: aspectos epidemiológicos Revista Brasileira de Medicina sporte. 7(1).

Oliveira, J. C. A., \& Tavares, D. M. S. (2010). Atenção ao idoso na estratégia de Saúde da Família: atuação do enfermeiro. Rev Esc Enferm USP; 44(3), 774-8. www.ee.usp.br/reeuspdf.

Sardinha-Peixoto, L., et al., Educação Permanente, continuada e em serviço: desvendando seus conceitos. Revista eletrônica trimestral de enfermagem. 2013. $29,324-340$.

Silva. M. F., et al. (2012). Relação entre os níveis de atividade física e qualidade de vida de idosos sedentários e fisicamente ativos. Rev. bras. Geriatria gerontologia. 15(4).

Siqueira, F. V. (2008). Atividade física em adultos e idosos residentes em áreas de abrangência de unidades básicas de saúde de municípios das regiões Sul e Nordeste do Brasil. Cad. Saúde Pública, Rio de Janeiro, 24(1),39-54.

Tannure, M. C. (2010). SAE: sistematização da assistência de enfermagem. Rio de Janeiro: Guanabara Koogan. 